\title{
A Dynamical Approach to Symplectic and Spectral Invariants for Billiards
}

\author{
Edoh Y. Amiran \\ Mathematics Department, Western Washington University, Bellingham, WA 98225-9063, USA
}

Received August 5, 1992

\begin{abstract}
The Lazutkin parameter for curves which are invariant under the billiard ball map is viewed symplectically in a way which makes it analogous to the sum of the values of a generating function over a closed orbit. This leads to relations among lengths of closed geodesics, lengths of invariant curves for the billiard map, rotation numbers, and the Lazutkin parameter. These relations establish the Birkhoff invariant and the expansion for the lengths of invariant curves in terms of the Lazutkin parameter as symplectic and spectral invariants (for the Dirichlet spectrum) and provide invariants which characterize a family of ellipses among smooth curves with positive curvature.

Geodesic flow on a bounded planar region gives rise to several geometric objects among which are closed reflected geodesics and invariant curves-closed curves whose tangents are invariant under reflection at the boundary. On a bounded domain, the map that assigns to each geodesic segment its successor after reflection at the boundary is called the billiard ball map and its dual (in the cotangent bundle for the boundary) is called the boundary map.

As shown by Guillemin and Melrose in [9], the lengths of closed geodesics are symplectic invariants associated with a generating function for the boundary map. Moreover, in a convex planar domain Marvizi and Melrose defined the wave invariants (see [12]), obtained via the interpolating hamiltonian of [14] which takes account of the singularity of the boundary map at the boundary. These wave invariants are symplectic and spectral invariants when viewed as functions of the rotation number associated to closed geodesics, but they do not have, as a whole, direct dynamical or geometric interpretations.

In this paper a new set of invariants, the caustics' invariants, are introduced. They are the lengths of invariant curves viewed as functions of the Lazutkin parameter of [11]. The Lazutkin parameter has a dynamical interpretation obtained by modifying a generating function for the boundary map.

Using these symplectic invariants one can isolate ellipses among planar domains whose curvature is strictly positive. In Sect. 6 it is shown that the caustics' invariants can be obtained from the wave invariants and the lengths of closed geodesics, and
\end{abstract}


that thus the caustics' invariants are also spectral invariants for the Dirichlet problem in the domain.

Functions with a dynamical nature defined in Sect. 4 on closed invariant curves supply the necessary connection with the symplectic setting described in the first two sections. The third section describes the caustics' invariants, and the fifth section shows that ellipses can be isolated using these invariants.

The author thanks John Sylvester for helpful conversations, especially regarding Sect. 5 .

\section{The Boundary Map and its Invariant Curves}

A geodesic in a bounded domain is a continuous curve consisting of line segments which meet at the boundary and obey the law of optic reflection there (successive segments make equal angles with the normal to the boundary).

Consider the circle bundle over the boundary, that is, $M=S_{\partial \Omega} R^{2} \subset T_{\partial \Omega} R^{2}$. The billiard ball map, $\beta$, is a map on the portion of $M$ pointing towards $\Omega$ taking $(x, u)$ to $(y, v)$, where $x$ and $y$ lie on the line generated by $u$, and $v$ is $u$ translated to $y$ and reflected w.r.t. the normal direction. Identifying the inward pointing portion of $S_{\partial \Omega} R^{2}$ (which we will denote $S_{\partial \Omega} \Omega$ ) with $B^{*} \partial \Omega$ the billiard ball map becomes the boundary map, $\delta$, on $B^{*} \partial \Omega$. It is well known that the boundary map preserves the natural 2-form on $B^{*} \partial \Omega$ (e.g. [4]).

An invariant section for $\delta$ is a smooth section $\xi: \partial \Omega \rightarrow B^{*} \partial \Omega$ with $\delta \circ \xi(\partial \Omega) \subset$ $\xi(\partial \Omega)$. It defines a collection of lines in the plane; for $x \in \partial \Omega,(x, w)=\xi(x)$ defines the line through $x$ along $w$. When $\partial \Omega$ is smooth and convex and the invariant curve is near $\partial \Omega$, these lines are the envelope of a smooth convex planar curve whose tangents remain invariant under optic reflection at $\partial \Omega$. (See, for example, [1].)

A planar curve, $C$, whose tangents are invariant under reflection at the boundary is called a caustic. It is clear that a line segment tangent to $C$ is part of a geodesic whose every segment is tangent to $C$ and thus that near $\partial \Omega$ there is a one-to-one correspondence of smooth convex caustics and invariant sections for the boundary map.

When it exists, the rotation number of the billiard ball map associated to $C$ is the average of the angle between successive line segments of a geodesic tangent to $C$, divided by $2 \pi$ (see also Sect. 4). For completeness we include

Lemma 1 [15]. Every geodesic tangent to a caustic has a unique rotation number and this rotation number is the same for all geodesics tangent to a fixed caustic.

Proof. Let $a, b \in C$ be two points on an oriented caustic. Let $p, q \in \partial \Omega$ be such that the forward tangents to $C$ at $a$ and $b$ intersect $\partial \Omega$ at $p$ and $q$ respectively. Since $C$ and $\partial \Omega$ are strictly convex, we can order $a \& b$ and $p \& q$ by their tangent angles. Since $C$ and $\partial \Omega$ are convex, $p \prec q$ iff $a \prec b$. Conversely, if $c, d \in C$ are such that the forward rays from $p, q \in \partial \Omega$ are tangent to $C$ at $c$ and $d$ respectively, then $c \prec d$ iff $p \prec q$. That is, the billiard ball map induces a monotone rotation on $C$.

Pick a geodesic, $g$, tangent to $C$. By the monotonicity of the rotation induced on $C$, the rotation number of $g$ is defined. Furthermore, if $g$ and $h$ are two distinct geodesics tangent to $C$, then every point of intersection of $h$, say, with $C$ lies between two points of intersection of $g$ with $C$, and thus by monotonicity the rotation number of $h$ is the same as the rotation number of $g$. 
For $C \subset \Omega$ a strictly convex curve and a point $q \in \partial \Omega$, there are precisely two points $a_{1}, a_{2} \in C$ so that the tangent lines to $C$ at $a_{1}$ and $a_{2}$ go through $q$ (see the figure in Sect. 3). We denote the lengths of the line segments between $a_{1}$ and $q$ and between $q$ and $a_{2}$ by $r$ and $l$, and the arclength along $C$ between $a_{1}$ and $a_{2}$ by $s$, and define the Lazutkin parameter, $V$, of $C$ and $\partial \Omega$ at $q$ (or alternatively at $a_{1}$ ) by

$$
V(C, \partial \Omega, q)=r+l-s .
$$

Lemma 2 [11]. A strictly convex closed planar curve $C \subset \Omega$ is a caustic iff the Lazutkin parameter of $C$ and $\partial \Omega$ at $p \in \partial \Omega$ is independent of the point $p$. In fact,

$$
\frac{d V}{d u}(u)=\cos \theta_{+}(u)-\cos \theta_{-}(u),
$$

where $u$ denotes arclength along $\partial \Omega$ and $\theta_{+}(u)$ and $\theta_{-}(u)$ are the angles formed at $u$ with the tangent to $\partial \Omega$ by the outgoing and incoming geodesic segments.

This characterization of caustics will be used in what follows, and is central to our view of the billiard ball map and the boundary map.

\section{Generating Functions and Invariant Curves}

Here we follow the use of generating functions (see, for example, [3] p. 258) as in [9] to show that certain quantities are invariant under symplectic changes of coordinates. Let $\omega$ denote the symplectic form on $B^{*} \partial \Omega$, let $q$ denote the coordinate dual to arclength along $\partial \Omega, p$ denote the orthogonal canonical coordinate $(\omega=d p \wedge d q)$, and let $\delta: B^{*} \partial \Omega \rightarrow B^{*} \partial \Omega$ denote the boundary map.

On $B^{*} \partial \Omega$ there is a one form $\alpha$ with $d \alpha=\omega$ and there is a function $G$ such that

$$
\delta^{*} \alpha-\alpha=d G
$$

with $G$ (normalized to be) zero on the boundary, $S_{+}^{*} \partial \Omega$. Since $B^{*} \partial \Omega$ corresponds to line segments in $\Omega, G$ can also be viewed as a function on $\partial \Omega \times \partial \Omega$.

While the generating function depends on the choice of $\alpha$, the sum over a closed orbit for $\delta$ does not; when $\left(x_{0}, x_{1}, \ldots, x_{k}\right)$ satisfy $\delta\left(x_{j}\right)=x_{\jmath+1}, j<k$ and $\delta\left(x_{k}\right)=x_{0}$ the sum

$$
L(x)=\sum_{j=0}^{k} G\left(x_{j}\right)
$$

is a symplectic invariant (see [9] p. 673). In our canonical coordinates as above $G\left(q_{1}, q\right)=\left|s_{1}-s\right|$ with $s$ and $s_{1}$ denoting the corresponding points on $\partial \Omega$ and distance computed in $\Omega \subset R^{2}$. Thus $L(x)$ is the length of the closed geodesic ([9] p. 676).

In terms of $\partial \Omega \times \partial \Omega$,

$$
\frac{\partial G\left(s_{1}, s\right)}{\partial s}=\cos \theta_{+}(s), \quad \text { and } \quad \frac{\partial G\left(s_{1}, s\right)}{\partial s_{1}}=-\cos \theta_{-}\left(s_{1}\right),
$$

where $\theta_{+}(s)$ and $\theta_{-}\left(s_{1}\right)$ are the angles formed by the line segment between $s$ and $s_{1}$ with $\partial \Omega$ at $s$ and at $s_{1}$ (in these coordinates $p(q)=\cos \theta_{+}(s)$ ).

Now let us return to an invariant curve $C$. This curve defines a section of $\partial \Omega \times \partial \Omega$ and also a section of $B^{*} \partial \Omega$. We denote the first by $C_{2}: s \mapsto t$, where $(s, t) \in \partial \Omega \times \partial \Omega$, with $s \prec t$ in the orientation of $\partial \Omega$, corresponds to a line segment 
tangent to $C$, and we denote the second by $C_{1}: s \mapsto p(s)$. Let $\pi: B^{*} \partial \Omega \rightarrow \partial \Omega$ be the natural projection and define $C_{B}: \partial \Omega \rightarrow B^{*} \partial \Omega$ by $C_{B}=C_{1} \circ C_{2}$.

With $t=C_{2}(s) \in \partial \Omega$ as before,

$$
\int\left[\cos \theta_{+}(t)-\cos \theta_{+}(s) \frac{d s}{d t}\right] d t=0
$$

because, over the closed boundary, the two parts of the integral are the same. It follows that there is an $f_{C}: \partial \Omega \rightarrow R$ with

$$
\frac{d f_{C}}{d t}=\cos \theta_{+}(t)-\cos \theta_{+}(s) \frac{d s}{d t} .
$$

What we have shown can be said invariantly; if $\alpha$ satisfies $d \alpha=\omega$ as above, then $\alpha$ restricts to a 1 -form on $C$ via the inclusion of $C$ in $B^{*} \partial \Omega$, and $f_{C}$ is a function on $C$ with

$$
d f_{C}=\left.\alpha\right|_{C}-\left.C_{B}^{*} \alpha\right|_{C} .
$$

Clearly, we can choose $f_{C}$ so as to depend smoothly on $C$, and satisfy $f_{\partial \Omega}=0$.

Finally, setting $g_{C}(t)=G(t, s)+f_{C}(t)$ we obtain from (2.1) and (2.2) that

$$
\frac{d g_{C}}{d t}(t)=\cos \theta_{+}(t)-\cos \theta_{-}(t) .
$$

In other words, $g_{C}(t)$ has a critical point at $t$ iff the segment from $C_{2}^{-1}(t)$ to $t$ remains tangent to $C$ after reflection at $t$.

As with the generating function, the sum of the values of $f_{C}$ over any closed orbit of $C_{2}$ is invariant under symplectic coordinate changes.

When $C$ is a caustic, a closed orbit tangent to $C$ is an orbit of $C_{2}$ and of $\delta$, and thus the sum of the values of $f_{C}$ and of $G$, and hence of $g_{C}$, over the orbit is a symplectic invariant. That is,

Theorem 3. When $\left(x_{0}, x_{1}, \ldots, x_{k}\right)$ satisfy $\delta\left(x_{j}\right)=x_{j+1}, j<k, \delta\left(x_{k}\right)=x_{0}$, and the segments $\pi\left(x_{\jmath}\right) \pi\left(x_{\jmath+1}\right)$ are tangent to $C$, the sum

$$
T(x)=\sum_{j=0}^{k} g\left(\pi\left(x_{j}\right)\right)
$$

is invariant under symplectic changes of coordinates.

From Lemma 2 it follows that the Lazutkin parameter defined at the end of the previous section satisfies (2.4). Clearly, if a closed geodesic tangent to a caustic $C$ has period $n$ then the value of the sum in Theorem 3 is $n V(C, \partial \Omega)$, where $V$ is the Lazutkin parameter of Sect. 1. We examine the consequences of this relation in Sect. 4.

\section{Caustics' Invariants}

Since $\partial \Omega$ has positive curvature, it can be parameterized by tangent angle. Choosing the counterclockwise orientation and the $(1,0)$ direction as the reference for measuring 
the tangent angle, there is a 1-1 correspondence between smooth strictly convex bounded regions and curvature functions, functions $k>0$ with period $2 \pi$ and

$$
\int_{0}^{2 \pi} k^{-1}(\theta) \cos \theta d \theta=\int_{0}^{2 \pi} k^{-1}(\theta) \sin \theta d \theta=0 .
$$

Let $w$ be the curvature of $\partial \Omega$ as a function of the tangent angle, $\phi$.

Let $C$ be a caustic for the billiard ball map on $\partial \Omega$, given by its curvature $k>0$, and with Lazutkin parameter $V$. We define the evolution operator,

$$
E: C^{\infty}(\mathbf{R} / 2 \pi \mathbf{Z}) \times \mathbf{R}^{+} \rightarrow C^{\infty}(\mathbf{R} / 2 \pi \mathbf{Z}),
$$

by $E(k, V)=w$.

A point $a$ on $C$, corresponding to tangent angle $\theta$, is given by

$$
a(\theta)=\left(x_{0}+\int_{0}^{\theta} \cos (t) \frac{d t}{k(t)}, y_{0}+\int_{0}^{\theta} \sin (t) \frac{d t}{k(t)}\right) .
$$

A point $q$ in $\partial \Omega$ is given by

$$
q(\phi)=a\left(\theta_{1}\right)+r\left(\cos \theta_{1}, \sin \theta_{1}\right)=a\left(\theta_{2}\right)-l\left(\cos \theta_{2}, \sin \theta_{2}\right),
$$

for some $\theta_{1}, r, \theta_{2}$, and $l$, and the Lazutkin parameter $V$ is

$$
V=r+l-\left(s\left(\theta_{2}\right)-s\left(\theta_{1}\right)\right) \text {, }
$$

where $s$ is the arclength along $C, s(\theta)=\int_{0}^{\theta} k^{-1}(t) d t$. (See the figure.)

Fig. 1. $\partial \Omega$ is the evolute of $\left(^{\prime}\right.$

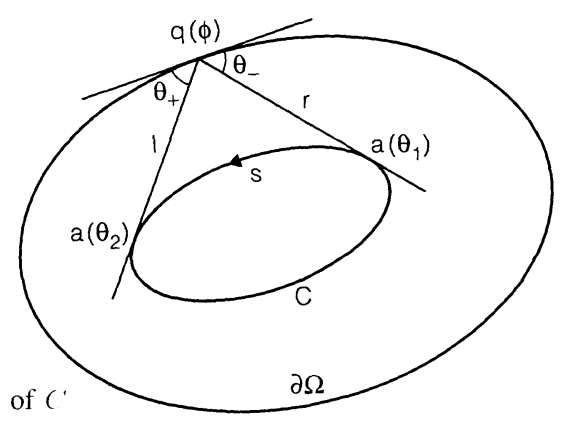

Since $C$ corresponds to an invariant curve for the billiard ball map on $\partial \Omega$,

$$
\phi\left(\theta_{1}+\theta_{2}\right) / 2 \text {. }
$$

A calculation of $r+l$ shows that

$$
V=\frac{1}{\cos \Delta} \int_{-\Delta}^{\Delta} \cos (u) k^{-1}(\phi+u) d u-\int_{-\Delta}^{\Delta} k^{-1}(\phi+u) d u
$$

where $\Delta=\theta_{2}-\phi=\phi-\theta_{1}$.

We have (see [1]) 
Proposition 4. $E(V, k)$ is a differential operator in $k$ which is smooth in $V^{2 / 3}$ for sufficiently small $V$.

The parameter $\Delta$ above is, essentially, the rotation number, $\eta$ (whose existence on $C$ follows from Lemma 1). More precisely,

$$
\frac{1}{2 \pi} \int_{0}^{2 \pi} 2 \Delta(\phi) d \phi=2 \pi \eta
$$

and

$$
\eta^{2} \sim(12)^{2 / 3} G(w) V^{2 / 3}+O\left(V^{4 / 3}\right), \quad G(w)=\int_{0}^{2 \pi} w^{2 / 3}(\phi) d \phi .
$$

Our variant of Birkhoff's invariant, which we call the scaling invariant, is $G(w)$.

After inversion and integration, we obtain a formal power series at $V=0$ for $L(C)$, the length of a caustic, in terms of $V^{2 / 3}$. The coefficients of this series are the desired caustics' invariants. That is, with $w$ and $k$ denoting the curvatures of $\partial \Omega$ and of $C$,

$$
w(\phi) \sim \sum_{j=0}^{\infty} E_{\jmath}(k)(\phi) V^{2 j / 3},
$$

and $E_{0}(k)=k$, so (formally) there are $M_{j}$ with

$$
k^{-1}(\phi) \sim \sum M_{j}(w)(\phi) V^{2 j / 3}
$$

and for the length of $C$

$$
L(C)=\int_{0}^{2 \pi} k^{-1}(\phi) d \phi \sim \sum_{j=0}^{\infty} J_{j} V^{2 j / 3}
$$

where

$$
J_{j}=J_{\jmath}(w)=\int_{0}^{2 \pi} M_{j}(w)(\phi) d \phi
$$

are the caustics' invariants. These invariants are easy to calculate from (3.1) and the geometry (see [1] where the $E_{j}$ are calculated). We will only need the first two caustics' invariant which are used in Sect. 5. Calculations give

$$
J_{0}=\int_{0}^{2 \pi} v^{-1}(\phi) d \phi=L(\partial \Omega), \quad J_{1}=-\left(\frac{3}{2}\right)^{2 / 3} \frac{1}{2} \int_{0}^{2 \pi} v^{-1 / 3}(\phi) d \phi
$$

and

$$
J_{2}=\frac{1}{720}\left(\frac{3}{2}\right)^{1 / 3} \int_{0}^{2 \pi}\left(8 v^{-5 / 3}\left(v^{\prime}\right)^{2}+9 v^{1 / 3}\right)(\phi) d \phi .
$$

Note that Lazutkin proved, using KAM theory and his parameter $V$, that there are uncountably many caustics in a sufficiently smooth convex planar domain, and that these caustics accumulate on the boundary, so that the expansions above can be obtained from the lengths of actual caustics. 


\section{Relations Arising From the Dynamics on a Caustic}

Consider a geodesic $\gamma$ (which we will view as a bi-infinite sequence in $S_{\partial \Omega} \Omega$ ) and a distinguished starting point $\xi$ in $\gamma$. Let $\angle\left(\beta^{n}(\xi), \beta^{n-1}(\xi)\right.$ ) denote the angle (in $T \mathbf{R}^{2}$ ) between the successive segments of the geodesic, and let $|$,$| denote distance in \mathbf{R}^{2}$. Set

$$
\eta_{m}(\gamma, \xi)=\frac{1}{m} \sum_{n=1}^{m} \angle\left(\beta^{n}(\xi), \beta^{n-1}(\xi)\right),
$$

and

$$
A_{m}(\gamma, \xi)=\frac{1}{m} \sum_{n=1}^{m}\left|\pi \circ \beta^{n}(\xi), \pi \circ \beta^{n-1}(\xi)\right|,
$$

where $\pi$ is the projection (to $\partial \Omega$ ).

On a caustic $C, A_{m}$ and $\eta_{m}$ are related to $V$ and $L(C)$ :

Proposition 5. For a caustic $C, \gamma$ a closed geodesic of period $m$ tangent to $C$, and $\xi \in \gamma$,

$$
m V(C, \partial \Omega)=m A_{m}(\gamma, \xi)-m \eta_{m}(\gamma, \xi) \cdot L(C),
$$

where $L(C)$ is the length of $C$.

Proof. This follows immediately from the definition of the Lazutkin parameter, $V$, after noticing that (with $\Delta$ as in Sect. 3)

$$
\angle\left(\beta^{n}(\xi), \beta^{n-1}(\xi)\right)=\Delta\left(\pi \beta^{n-1}(\xi)\right) .
$$

Proposition 6. For a geodesic $\gamma$ tangent to a fixed caustic $C$,

$$
\eta(\gamma):=\lim _{m \rightarrow \infty} \eta_{m}(\gamma, \xi)
$$

is well defined. In fact, $\eta$ is independent of the choice of $\gamma$ tangent to $C$.

Proof. By Lemma 1 the billiard ball map restricted to $C$ has a well defined rotation number so the limit above converges for any $\xi$ and is independent of $\xi$. By Lemma 1 , this limit is also independent of $\gamma$.

Corollary 7. For a geodesic $\gamma$ tangent to a caustic $C$,

$$
A(\gamma):=\lim _{m \rightarrow \infty} A_{m}(\gamma, \xi)
$$

is well defined and independent of $\gamma$. Moreover,

$$
V(C, \partial \Omega)=A(\gamma)-\eta(\gamma) L(C)
$$

Theorem 8. The caustics' invariants are invariant under symplectic changes of coordinates.

Proof. Fix a caustic $C$ and a geodesic $\gamma$ tangent to it. For any $\xi \in \gamma$ and positive integer $p$, the least positive $n$ with

$$
\sum_{i=1}^{n} \angle\left(\beta^{i}(\xi), \beta^{i-1}(\xi)\right)>2 \pi p
$$


is unchanged by symplectic coordinate changes because a symplectic map preserves the boundary and the ordering of points on the boundary. Thus the rotation number, $\eta(\gamma)$, is a symplectic invariant.

Similarly, $A(\gamma)$ can be approximated by the averaged lengths of closed geodesics. Since the lengths of these geodesics are invariant under symplectic coordinate changes, and since the period of each geodesic is a symplectic invariant as noted in the previous paragraph, $A(\gamma)$ is a symplectic invariant.

By Theorem 3, the calculation following it, and the invariance of the period, $V(C)$ is a symplectic invariant. It follows from $(4.1)$ that $L(C)$ is a symplectic invariant and then that the coefficients in the expansion of $L(C)$ in powers of $V(C)^{2 / 3}$ are symplectic invariants.

\section{Extrema and Ellipses}

In this section we wish to investigate the relations of the invariants

$$
L(k)=J_{0}=\int_{0}^{2 \pi} k^{-1}(\theta) d \theta, \quad J_{1}(k)=\int_{0}^{2 \pi} k^{-1 / 3}(\theta) d \theta,
$$

and

$$
\left.G(k)=\int_{0}^{2 \pi} k^{2 / 3}(\theta) d \theta\right)
$$

where $k>0$ is the curvature of the boundary of a planar domain given as a function of tangent angle $\theta$. (The invariants $J_{0}$ and $J_{1}$ are also wave invariants as defined in [12].)

Since $k$ represents a closed curve it satisfies

$$
\int_{0}^{2 \pi} \cos (\theta) k^{-1}(\theta) d \theta=\int_{0}^{2 \pi} \sin (\theta) k^{-1}(\theta) d \theta=0 .
$$

Consider those smooth closed curves with positive curvature and with a fixed length, $L$. We will examine such curves which are critical points for $G$.

It is clear that $G$ is non-negative (since $k>0$ ), and Hölder's inequality, with $1=k^{2 / 5} \cdot k^{-2 / 5}$, gives

$$
2 \pi \leq\left(\int_{0}^{2 \pi} k^{2 / 3}\right)^{3 / 5}\left(\int_{0}^{2 \pi} k^{-1}\right)^{2 / 5}=G^{3 / 5} L^{2 / 5}
$$

which shows that $G$ is positive with an absolute minimum only when $k$ is constant (and the region is a disk).

Let us, nonetheless, consider a variant of $G$. First set $r=k^{-1}$, and then note that, since the integral of an exact derivative is zero,

$$
\int_{0}^{2 \pi}\left(r^{-5 / 3} r^{\prime \prime}-\frac{5}{3} r^{-8 / 3}\left(r^{\prime}\right)-6 r^{-2 / 3}\right)(\theta) d \theta=-6 G(r) .
$$


Letting $\varepsilon u$ be a small smooth periodic variation in $r$ (with $\varepsilon \rightarrow 0$ ), $r$ is critical for $-6 G$ with $L$ fixed only if for any $u$

$$
\int u(\theta) d \theta=0 \Rightarrow \int\left(r^{-5 / 3} r^{\prime \prime}-\frac{5}{3} r^{-8 / 3}\left(r^{\prime}\right)^{2}-6 r^{-2 / 3}\right) u=0 .
$$

Since $u$ is periodic, the first of these equations reduces to $u=d f / d \theta$, for some smooth periodic $f$. The condition (5.2) becomes

$$
\int\left(r^{-5 / 3} r^{\prime \prime}-\frac{5}{3} r^{-8 / 3}\left(r^{\prime}\right)^{2}-6 r^{-2 / 3}\right)^{\prime} f=0
$$

for any smooth periodic $f$. Hence $r$ must satisfy

$$
r^{-5 / 3} r^{\prime \prime}-\frac{5}{3} r^{-8 / 3}\left(r^{\prime}\right)^{2}-6 r^{-2 / 3}=\text { constant. }
$$

The solutions to this equation are precisely ellipses and their rotations.

The condition (5.3) is not sufficient to determine a critical point of $-6 G$. The radius of curvature of an ellipse with major axis $2 a$ and minor axis $2 b$ is

$$
e(\theta)=(a b)^{2}\left[a^{2}(\cos \theta)^{2}+b^{2}(\sin \theta)^{2}\right]^{-3 / 2},
$$

and then

$$
G(e)=\pi(a b)^{-4 / 3}\left(a^{2}+b^{2}\right) .
$$

Thus, an ellipse maximizes $-6 G$ among variations which fix the length $(L)$ and are also transversal to ellipses, but only circles are an absolute minimum among the family of ellipses with length $L$.

Using (5.4) one can check directly that the conditions on a family of ellipses for fixing $L$ and for fixing $J_{1}$ are different. In fact, $L$ is fixed only if

$$
\begin{aligned}
\frac{d b}{d a}= & -\frac{b}{a} \int_{0}^{2 \pi}\left(2 b^{2} \sin ^{2}(\theta)-a^{2} \cos ^{2}(\theta)\right)\left[a^{2} \cos ^{2}(\theta)+b^{2} \sin ^{2}(\theta)\right]^{-5 / 2} d \theta \\
& \div \int_{0}^{2 \pi}\left(2 a^{2} \cos ^{2}(\theta)-b^{2} \sin ^{2}(\theta)\right)\left[a^{2} \cos ^{2}(\theta)+b^{2} \sin ^{2}(\theta)\right]^{-5 / 2} d \theta
\end{aligned}
$$

while $J_{1}$ is fixed only if

$$
\begin{aligned}
\frac{d b}{d a}= & -\frac{b}{a} \int_{0}^{2 \pi}\left(2 b^{2} \sin ^{2}(\theta)-a^{2} \cos ^{2}(\theta)\right)\left[a^{2} \cos ^{2}(\theta)+b^{2} \sin ^{2}(\theta)\right]^{-3 / 2} d \theta \\
& \div \int_{0}^{2 \pi}\left(2 a^{2} \cos ^{2}(\theta)-b^{2} \sin ^{2}(\theta)\right)\left[a^{2} \cos ^{2}(\theta)+b^{2} \sin ^{2}(\theta)\right]^{-3 / 2} d \theta
\end{aligned}
$$
that is

Thus each ellipse maximizes $-6 G$ among curves with a fixed value of $L$ and $J_{1}$,

Theorem 9. Assume that a planar curve is smooth, closed, has strictly positive curvature, has fixed first wave or caustics' invariant (the length $L$ ) and second wave or caustics' invariant $\left(J_{1}\right)$, and maximizes $-6 G$, where $G$ is the scaling invariant. Then the curve is an ellipse. 


\section{Spectral Invariance}

We return to the setting of Sect. 2

Marvizi and Melrose (see [12]) showed that their wave invariants (recalled below) can be obtained from the spectrum of the Laplacian in $\Omega$ with Dirichlet (or Neumann or Robin) boundary conditions. Through the use of the interpolating hamiltonian they calculate the one-form $\alpha$ and consequently determine an expression, which involves the wave invariants, for the generating function, $G$. They show that the length spectrum (the collection of sums of $G$ over closed orbits) is spectrally invariant, and finally, that the power series resulting for the wave invariants can be recovered from the spectrum.

The coordinates introduced in [12] are suitable for analyzing the expression in Eq. (2.3), and so we use their construction.

Let $\omega$ denote the symplectic form on $B^{*} \partial \Omega, s$ denote arclength, and let $\delta$ : $B^{*} \partial \Omega \rightarrow B^{*} \partial \Omega$ denote the boundary map. Then there is a function

$$
\zeta \in C^{\infty}\left(B^{*} \partial \Omega\right) \text { with } \delta=\exp \left(-\zeta^{1 / 2} H_{\zeta}\right)+\psi
$$

where $H_{\zeta}$ is the Hamiltonian vector field of $\zeta$, and $\psi$ is a smooth symplectic map which fixes the boundary component $S_{+}^{*} \partial \Omega$ of $B^{*} \partial \Omega$ to all orders. This determines the Taylor series for $\zeta$ at $S_{+}^{*} \partial \Omega$. (The hamiltonian above is called the interpolating hamiltonian-see [14].) One can choose $\zeta$ so that $d \zeta \neq 0$ on all of $B^{*} \partial \Omega$ and so that $\zeta=2$ on the boundary component $S_{-}^{*} \partial \Omega$.

The one-form $d z$ is defined on the curve $\{\zeta=c\}$ via $d z\left(H_{\zeta}\right)=1$ and $z(s=0)=0$, and the function

$$
I(c)=\int_{\{\zeta=c\}} d z
$$

is $C^{\infty}$ in $c$ near 0 , with a Taylor series at $c=0$ which is independent of the choice of $\zeta$. The wave invariants are the coefficients in this Taylor series, namely

$$
I_{k+1}=\frac{d^{k} I}{d c^{k}}(0), \quad k=0,1,2, \ldots
$$

Subsequently, [12] shows that

$$
\delta^{*} z \equiv z-\zeta^{1 / 2}, \quad \delta^{*} \zeta \equiv \zeta, \quad \text { and } \quad \omega=d \zeta \wedge d z
$$

where equivalence is in the sense of Taylor series at $\zeta=0$. They now set

$$
F(\zeta)=\int_{0}^{\zeta} I(u) d u, \quad \text { and } \quad \alpha=F(\zeta) d(z / I(\zeta))+d s
$$

Then $\alpha$ is a 1 -form on $B^{*} \partial \Omega$ with $d \alpha=\omega$.

Further calculations show that

$$
\delta^{*} \alpha-\alpha=d\left(-\frac{F(\zeta)}{I(\zeta)} \zeta^{1 / 2}+\frac{2}{3} \zeta^{3 / 2}+\delta^{*} s-s+h\right),
$$

where $h$ vanishes to all orders at $S_{+}^{*} \partial \Omega$. This determines the generating function, $G$, explicitly. 
Marvizi and Melrose then observe that on a simple closed geodesic with period $n$, $\gamma_{1, n}$,

$$
I(\zeta) \equiv n \zeta^{1 / 2}
$$

and thus the length of $\gamma_{1, n}$ is

$$
L\left(\gamma_{1, n}\right)=L(\partial \Omega)-F(\zeta)+\frac{2}{3} \zeta I(\zeta) .
$$

They now conclude that $\zeta$ and $I(\zeta)$, computed for closed simple geodesics, have asymptotic expansions in terms of the rotation number squared, that is $1 / n^{2}$ for $\gamma_{1, n}$.

Differentiating (6.4) and (6.3) (implicitly), one concludes that the power series for $\zeta$ and $I(\zeta)$ as functions of the rotation number (squared) are determined by the length spectrum.

Under the non-coincidence assumption (see p. 490 of [12]), that for sufficiently large periods the length of no closed non-simple geodesic coincide with the infimum (or sumpremum) of the lengths of simple closed geodesics with each fixed period, the length spectrum is determined by the spectrum for the Laplacian with Dirichlet or Neumann or Robin boundary conditions (see [9] and [12]); the asymptotics above are spectral invariants.

But on a caustic, $C, \zeta$ is constant, so $\left.d \zeta\right|_{C}=0$ and

$$
\left.\alpha\right|_{C}-\left.C_{B}^{*} \alpha\right|_{C}=d\left(s-C_{B}^{*} s\right) .
$$

Consequently

$$
V(C, \partial \Omega)=-\zeta^{1 / 2} \frac{F(\zeta)}{I(\zeta)}+\frac{2}{3} \zeta^{3 / 2}
$$

The expansions, in terms of the rotation number, for $\zeta$ and $I(\zeta)$ are determined by the spectrum and $F(\zeta)$ is defined in terms of these - by (6.2). Therefore, the expansion for $V(C, \partial \Omega)$ is determined by the spectrum, and it follows immediately from Proposition 5 (in Sect. 4) that

Theorem 10. The caustics' invariants defined by (3.1) are determined by the spectrum of the Laplacian in a planar domain that satisfies the non-coincidence condition.

Corollary 11. Ellipses are determined by the spectrum of the Laplacian among smooth planar domain whose boundary has strictly positive curvatures and which satisfy the non-coincidence condition.

Remark. The first heat invariant, which is spectrally determined, is the area. Thus the parameters for an ellipse can be calculated directly from this invariant, $(\pi a b)$ for the ellipse with axes $2 a$ and $2 b$, and the scaling invariant (5.5).

\section{References}

1. Amiran, E.Y.: Caustics and evolutes for convex planar domains. J. Diff. Geom. 28, 345-357 (1988)

2. Andersson, K.G., Melrose, R.B.: The propagation of singularities along gliding rays. Invent. Math. 41, 23-95 (1977)

3. Arnold, V.I.: Mathematical Methods of Classical Mechanics. Berlin, Heidelberg, New York: Springer 1980

4. Birkhoff, G.D.: On the periodic motions of dynamical systems. Acta Math. 50, 359-379 (1927). Reprinted in Collected Mathematical Papers, vol II, Providence, RI: Am. Math. Society, 1950, pp. $111-229$ 
5. Chazarin, J.: Formule de Poisson pour les variétés Riemanniennes. Invent. Math. 24, 65-82 (1974)

6. Colin de Verdière, Y.: Spectre du Laplacian et longueurs des géodésiques périodiques II. Comp. Math. 27, 159-184 (1973)

7. Duistermaat, J.J., Guillemin, V.W.: The spectrum of positive operators and periodic geodesics. Invent. Math. 29, 29-79 (1975)

8. Guillemin, V.W., Melrose, R.B.: The Poisson summation formula for manifolds with boundary. Adv. in Math. 32, 204-232 (1979)

9. Guillemin, V.W., Melrose, R.B.: A cohomological invariant of discrete dynamical systems. In: Butzer, P.L. and Feher, F. (eds), Christoffel Symposium 1979, Basel: Birkhäuser, 1981

10. Kac, M.: Can one hear the shape of a drum? Am. Math. Soc. Monthly 73 (II), 1-23 (1966)

11. Lazutkin, V.F.: Existence of a continuum of closed invariant curves for a convex billiard. Math. USSR Izvestija 7, no. 1, 185-214 (1973)

12. Marvizi, S., Melrose, R.B.: Spectral invariants of convex planar regions. J. Differ. Geom. 17, 475-502 (1982)

13. McKean, H.P., Singer, I.M.: Curvature and the eigenvalues of the Laplacian. J. Differ. Geom. 1, 43-69 (1967)

14. Melrose, R.B.: Equivalence of glancing hypersurfaces. Invent. Math. 37, 165-191 (1976)

15. Poincaré, H.: Sur un théorèm de géométrie, Randiconti del Circolo Mathematico di Palermo, vol. 33, 1912

16. Osserman, R.: The isoperimetric inequality. Bull. Am. Math. Soc. 84, 1182-1238 (1978)

Communicated by Ya. G. Sinai 